\title{
Fuzzy Coloring and Fuzzy Chromatic Number of Matching and Perfect Matching Using Alpha Cut In Unary Operations
}

\author{
S. Yahya Mohamad ${ }^{1}$ and S.Suganthi ${ }^{2}$ \\ ${ }^{I} P G \&$ Research Department of Mathematics, Government Arts College, Trichy-22, Tamilnadu, India. \\ Email: yahya-md@yahoo.com \\ ${ }^{2}$ Department of Mathematics,Bharathiyar Arts \& Science College for Women, Deviyakurichi, Tamilnadu, India. \\ Email: sivasujithsuganthi@gmail.com
}

\begin{abstract}
A graph is said to be a complete fuzzy labeling graph if it has every pair of adjacent vertices of the fuzzy graph. A matching is a set of non-adjacent edges. If every vertex of fuzzy graph is M-saturated then the matching is said to be complete or perfect. In this paper, we introduced the new concept of fuzzy coloring and fuzzy chromatic number using $\propto$ cut through matching and complete matching in Unary operations. We iscussed some properties using these concepts.
\end{abstract}

Keywords: Matching, perfect matching, $\propto$ cut, Fuzzy coloring, Fuzzy chromatic number.

AMS Mathematics Subject Classification (2010): 05C72, 54E50, 03F55

\section{INTRODUCTION}

Graph theory is rapidly moving into mainstream of mathematics mainly because of its applications in diverse fields with include biochemistry (DNA double helix and SNP assembly Problem), chemistry (model chemical compounds) electrical engineering(communication networks and coding theory) computer science (algorithms and computations) and Operations Research (scheduling)[12]. Graph coloring is one of the most important concepts in Graph Theory and is used in many real time applications like Job scheduling, Aircraft scheduling, computer network security ,Map coloring and GSM mobile phone networks etc.

A graph coloring is the assignment of color to each vertex of the graphs so that no two adjacent vertices are assigned the same color. Similarly for the assignment of color to each edge of the graphs so that no two incident edges are assigned the same color. The minimum number of colors needed to color a graph is called its chromatic number and it is denoted by $\chi(\mathrm{G})[9]$. Many Problems of practical interest that can be modeled as graph theoretic problems may be uncertain. To deal with this uncertainty the concept of fuzzy theory was applied to graph theory[12].

A fuzzy set was defined by L. A. Zadeh[19] in 1965.Every element in the universal set is assigned a grade of membership ,a value in $[0,1]$.The elements in the universal set along with their grades of membership form a fuzzy set. In 1965 Fuzzy relations on a set was first defined by Zadeh. Among many branches of modern mathematics, the theory of sets (which was founded by G.Cantor occupies a unique place. The mathematical concept of a set can be used as foundation for many branches of modern mathematics[15].
A graph is said to be a complete fuzzy labeling graph if it has every pair of adjacent vertices of the fuzzy graph. A matching is a set of non-adjacent edges. If every vertex of fuzzy graph is M-saturated then the matching is said to be complete or perfect. In this paper, we introduced the new concept of fuzzy coloring and fuzzy chromatic number using $\propto$ cut through matching and complete matching in Unary operations. We discussed some properties using these concepts.

\section{PRELIMINARIES} Definition: 2.1

A graph with $n$ vertices in which every pair of distinct vertices is joined by a line is called complete graph on $\mathrm{n}$ vertices. It is denoted by $\mathrm{K}_{\mathrm{n}}$. Definition 2.2

Let $\mathrm{U}$ and $\mathrm{V}$ be two sets. Then $\rho$ is said to be a fuzzy relation from $\mathrm{U}$ into $\mathrm{V}$ if $\rho$ is a fuzzy set of $\mathrm{U} \times \mathrm{V}$. A fuzzy graph $\mathrm{G}=(\alpha, \beta)$ is a pair of functions $\alpha: \mathrm{V} \rightarrow[0,1]$ and

$\beta: \mathrm{V} \times \mathrm{V} \rightarrow[0,1]$ where for all $\mathrm{u}, \mathrm{v} \in \mathrm{V}$, we have $\beta$ (u ,v) $\leq \min \{\alpha(\mathrm{u}), \alpha(\mathrm{v})\}$.

\section{Definition 2.3}

A graph $G=(\alpha, \beta)$ is said to be a fuzzy labeling graph if $\alpha: \mathrm{V} \rightarrow[0,1]$

and $\beta: \mathrm{V} \times \mathrm{V} \rightarrow[0,1]$ is a bijective such that the membership value of edges and vertices are distinct and $\beta(\mathrm{u}, \mathrm{v})<\min \{\alpha(\mathrm{u}), \alpha(\mathrm{v})\}$ for all $\mathrm{u}, \mathrm{v}$ $\in \mathrm{V}$.

Example: 2.4 


\section{Available online at www.ijrat.org}

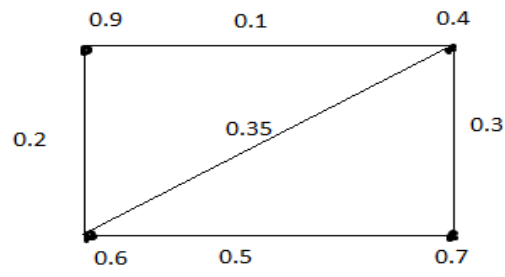

Definition: 2.5 .

A fuzzy graph $\mathrm{G}=(\alpha, \beta)$ is said to be complete if $\beta(\mathrm{u}, \mathrm{v})=\min \{\alpha(\mathrm{u}), \alpha(\mathrm{v})\}$ for all $\mathrm{u}, \mathrm{v} \in$ $\mathrm{V}$ and every pair of vertices are adjacent. It is denoted by $\mathrm{K}_{\mathrm{n}}[\mathrm{FLG}]$.

\section{Example 2.6}

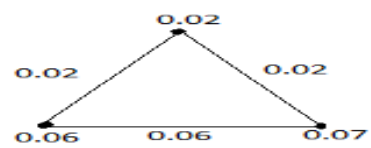

\section{Definition: 2.7}

A subset $M$ of $\beta\left(v_{i, v i+1}\right), 1 \leq i \leq n$ is called a matching in fuzzy graph

if its elements are links and no two are adjacent in G. The two ends of an edge in $M$ are

Said to be matched under M.

Example: 2.8

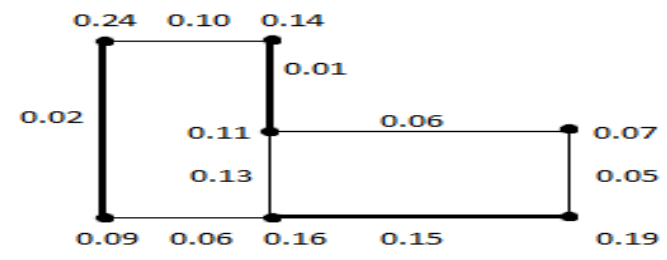

Definition 2.9

If every vertex of fuzzy graph is Msaturated then the matching is said to be complete or perfect. It is denoted by $\mathrm{C}_{\mathrm{M}}$.

Example: 2.10

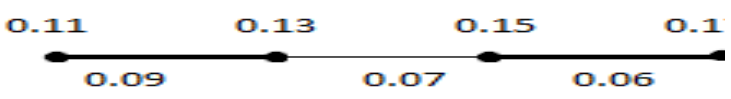

Definition 2.11

The degree of the vertex in a graph $\mathrm{G}$ is defined to be the number of vertices adjacent to that vertex .Here the maximum degree is denoted by $\Delta$ and minimum degree is denoted by $\delta$.

Definition: $\mathbf{2 . 1 2}$

A family $\lambda=\left\{\mathrm{M}_{1}, \mathrm{M}_{2}, \mathrm{M}_{3} \ldots \ldots \ldots . \mathrm{M}_{\mathrm{k}}\right\}$ of fuzzy sets on a set $E$ is called a M- fuzzy coloring of $G=(V$, $\alpha, \beta)$ if

(i) $\quad \vee \lambda=\beta$. It means no edge belongs to two different color classes.

(ii) $\quad \lambda_{\mathrm{i}} \Lambda \lambda_{\mathrm{j}}=0$.

(iii) For every effective edge $(\mathrm{x}, \mathrm{y})$ of $\mathrm{G}$ $\min \left\{\lambda_{\mathrm{i}}(\mathrm{x}), \lambda_{\mathrm{j}}(\mathrm{y})\right\}=0(1 \leq \mathrm{i} \leq \mathrm{k})$. (This means any one of the edges does not receive different color).

The minimum number $\mathrm{k}$ for which there exists a M-fuzzy coloring is called fuzzy matching chromatic number. It is denoted by $\chi^{\mathrm{fm}}(\mathrm{G})$.

\section{MAIN RESULTS}

Unary operation is an operation in which a new graph is obtained from old one by applying some alterations. Here we discussed fuzzy coloring and fuzzy chromatic number of matching and perfect matching using alpha cut in some unary operations like
(i) Line graph
(ii) Power graph
(iii) Medial graph
(iv) Dual graph

\section{Definition :3.1}

The $\alpha$-cut respect to the matching $\mathrm{A}_{\alpha}$ $(\mathrm{M})=\{\alpha \in M / \mu A(M) \geq \alpha\}$.Here $\alpha$-cut depends on edge membership value and $0 \leq \alpha \leq 1$.

\section{Definition :3.2}

The line fuzzy graph LFG is a new graph obtained from initial one fuzzy Graph FG in which the vertices are the edges of FG and the corresponding vertices adjacent only when they have common vertex as its adjacency.

Example

(i)

$$
\mathrm{V}_{1}(0.2) \quad \mathrm{V}_{1}(0.2)
$$

$$
\mathrm{K}_{1} \quad \mathrm{~L}\left(\mathrm{~K}_{1}\right)
$$

Here matching not exists. Hence Chromatic Number $\chi M$ (LK1) not exists.

(ii)

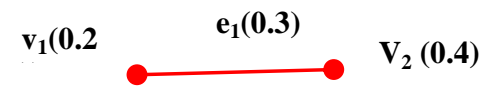

\section{$\mathbf{K}_{2}$}

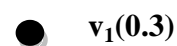

\section{$\mathrm{L}\left(\mathrm{K}_{2}\right)$}

Suppose we take $\alpha=0.3$. Here Perfect matching $\mathrm{M}=\{0.3\}$

Here the number of colors required to color the matching is 1.Hence $\chi M\left(\mathrm{~K}_{2}\right)=1$ and $\chi M\left(\mathrm{LK}_{2}\right)$ not exists.

Suppose we take $\alpha=0.4$.Here edge not exists. Hence $\chi M\left(\mathrm{~K}_{2}\right)$ and $\chi M\left(\mathrm{LK}_{2}\right)$ not exists.

(iii)

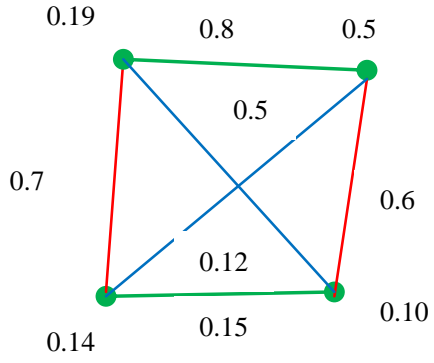




\section{Available online at www.ijrat.org}

$\mathrm{K}_{4}$

Here the perfect matching $\mathrm{M}_{1}=\{0.8,0.15\}$, $\mathrm{M}_{2}=\{0.7,0.6\}, \mathrm{M}_{3}=\{0.5,0.12\}$.

Hence $\chi M 1\left(\mathrm{~K}_{4}\right)=1, \chi M 2\left(\mathrm{~K}_{4}\right)=1$ and $\chi M 3\left(\mathrm{~K}_{4}\right)=1$

$\mathbf{v}_{1}(\mathbf{0 . 8})$

$\mathbf{V}_{4}(\mathbf{0 . 7})$

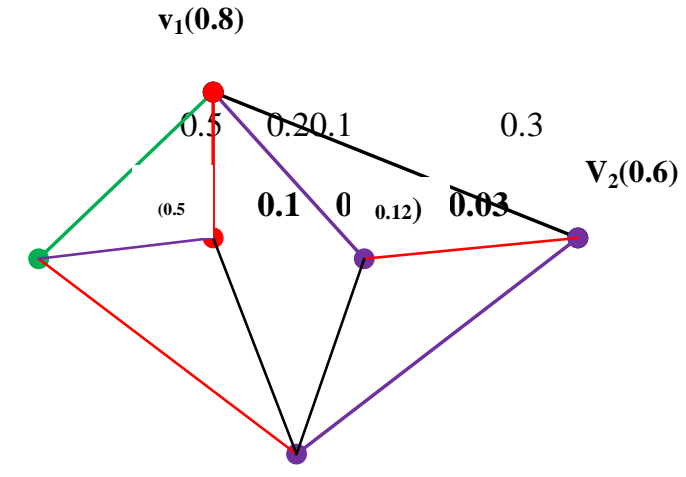

$\mathrm{V}_{3(\mathbf{0 . 1 5})}$

LK4

In $\mathrm{K}_{4}$, For any $\alpha$-cut $\chi M 1\left(\mathrm{~K}_{4}\right) 1, \chi M 2\left(\mathrm{~K}_{4}\right)$ and $\chi M 3\left(\mathrm{~K}_{4}\right)$ are either $\mathbf{0}$ or 1.

In $\mathrm{LK}_{4}$, For any $\alpha$-cut $\chi M\left(\mathrm{~K}_{4}\right)$ are either $\mathbf{0}$ or 1. Here only two perfect matching exists.

Note:

Line graph of a complete graph need not be complete.

\section{Theorem:3.3}

For any value of $\alpha$-cut, matching and perfect matching of the fuzzy labeling graph and its line graph has always the chromatic number either zero or one.

Proof

Consider fuzzy graph FG and its line graph LFG. Also we take the matching and perfect matching for the respective graphs.

We take $\alpha$-cut as the minimum membership value of the edge in the matching(perfect matching).

Suppose the membership value of the edge in the matching(perfect matching) is greater than or equal to $\alpha$-cut then we keep the same edges in the matching.

If not, we delete the corresponding edges in the matching. But matching means the set of non adjacent edges. So all the edges in the matching received same color. Hence the chromatic number is one. According to the value of $\alpha$-cut, if there exists no edges in the matching then the matching received no color. Hence the chromatic number is zero.

Hence for any value of $\alpha$-cut, matching and perfect matching of the fuzzy labeling graph and its line graph has always the chromatic number either zero or one.

\section{Definition :3.4}

The $\mathrm{n}^{\text {th }}$ power of the fuzzy labeling graph is the fuzzy labeling graph which contains same number of vertices in $\mathrm{G}$ and in which any two vertices are adjacent only when the length of the shortest path joining these two vertices is atmost $n$.

\section{Example}

(i)

$$
\mathrm{V}_{1}(0.2)
$$

FG

(FG) ${ }^{2}$

Here matching not exists. Hence Chromatic Number $\chi M\left((\mathbf{F G})^{2}\right)$ not exists.

(ii)
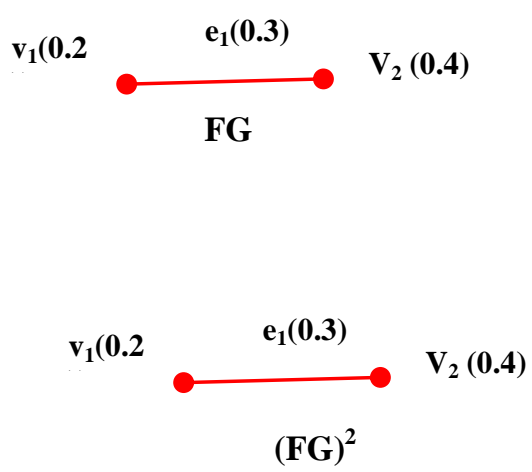

Suppose we take $\alpha=0.3$.Here Perfect matching $\mathrm{M}=\{0.3\}$

Here the number of colors required to color the matching is 1 .Hence $\chi M(\mathrm{FG})=1$ and $\chi M(\mathrm{FG})^{2}$ not exists.

Note:

Any power of $\mathrm{K}_{1}$ and $\mathrm{K}_{2}$ are always same.

(iii)
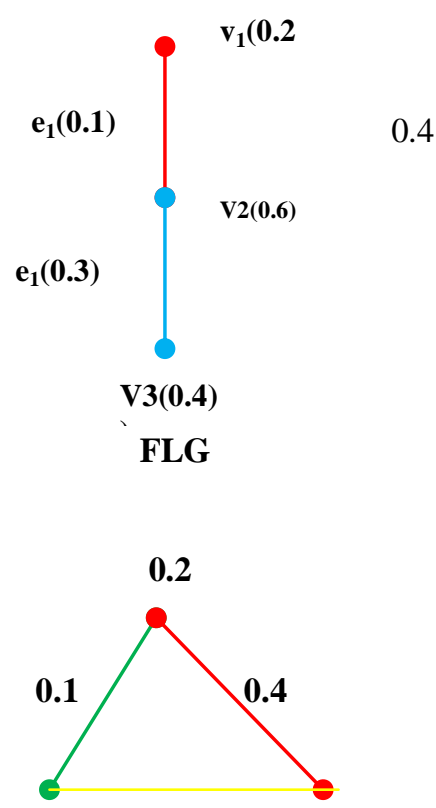


\section{Available online at www.ijrat.org}

$\begin{array}{lll}0.6 & 0.3 & 0.4 \\ & \left(\mathbf{K}_{\mathbf{3}}\right)^{2} & \end{array}$

Number of matching in FLG is 2 and number of matching in any power of $\mathrm{K}_{3}$ is 3 .

(iv)

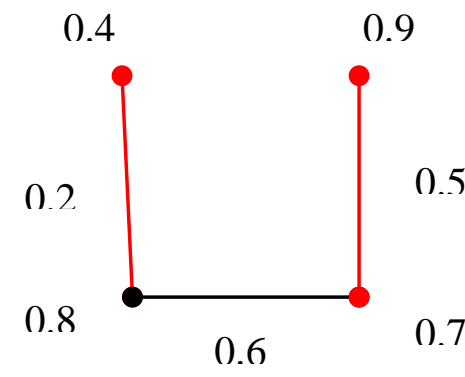

FLG

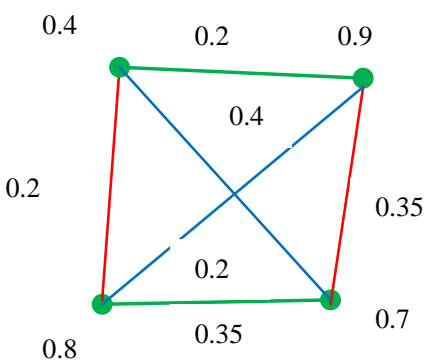

$\left(\right.$ FLG) ${ }^{2}$

Number of perfect matching in FLG is 1 and number of matching in any power of $\mathrm{K}_{4}$ is 3 .

Note :

If we get complete graph in $\mathrm{K}^{\text {th }}$ power with any number of vertices then the power of $(\mathrm{K}+1) .(\mathrm{K}+2) \ldots .$. is also complete.

\section{Definition: 3.5}

A fuzzy graph drawn in any surface is called fuzzy plane graph. A fuzzy planar graph is a fuzzy graph in which the edges are not intersect. The fuzzy planar graph divide the plane into region is called the Faces .

Definition: 3.6

The number of edges in the face is called the cardinality of the face.

Definition: 3.7

The fuzzy Medial graph is a fuzzy graph which contains the vertices (which are the edges of fuzzy graph) and the adjacency between the vertices exists only when the edges occur consecutively in the faces.

Definition: 3.8

The fuzzy Dual graph is also a fuzzy graph that has vertex for each face of FG and there exists an edge whenever two faces of FG are separated from each other by an edge and self-loop exists when the same face appears on both sides of an edge.

Note
The degree of the vertex inside the faces in fuzzy Dual graph is equal to the cardinality of the corresponding faces.

\section{CONCLUSION}

In this paper, we introduced the new concept of fuzzy coloring and fuzzy chromatic number for some special fuzzy graphs like line graph, power graph, medial graph and Dual Graph through matching and complete matching using $\alpha$-cut and we discussed some properties using these concepts. In future we extend this concept to binary operations.

\section{REFERENCES}

[1] Anjaly Kishore, M.S. Sunitha,Chromatic Number of Fuzzy Graph, Annals of Fuzzy Mathematics and Informatics, Vol 10,2013.

[2] Arindam Dey,Dhrubajyoti Ghosh ,Anita Pal , Edge Coloring of a Complement Fuzzy Graph, International journal of Modern Engineering Research, Vol 22012.

[3] Frank Harary, Graph Theory, Indian Student Edition, Narosa/Addison Wesley, 1988.

[4] Jahir Hussain R , Kanzul Fatima K S, Fuzzy Chromatic Number of Middle ,Subdivision and Total Fuzzy Graph,International Journal of Mathematical Archieve-6(12),2015.

[5] Jemal Abawajy,AndrevKelarev ,Morshed Chowdhury,Power Graph Survey,Electronic journal of Graph theory and Applications1(2)2013,125-147.

[6] Narsingh Deo, Graph Theory with applications to Engineering and Computer Science.

[7] Nagoorgani.A and V. T. Chandrasekaran V. T, First Look at Fuzzy Graph Theory, Allied Publishers, 2010.

[8] Nagoorgani. A and Latha S. R., Some operations on fuzzy graph, Proceedings of the International Conference on Mathematical Methods and Computation, Feb.2014.

[9] Nivethana.V, Parvathi.A, Fuzzy Total Coloring and Chromatics Number of a Complete Fuzzy Graph, International journal of Emerging Trends in Engineering and Development, Vol6,2013.

[10] Ranganathan K , Balakrishnan R, A Text Book of Graph Theory, Springer.

[11] Rosenfeld A, Fuzzy graphs, Fuzzy Sets and their Applications to Cognitive and Decision Process, L. A. Zadeh, K. S. Fu, K. Tanaka and M. Shimura, eds., Academic Press, New York, 1975, pp. 75-95.

[12] Dr. Savithri D, Gayathri devi A,Application of Alpha cut coloring of a fuzzy graph , International Journal of computer engineering and technology, Vol 5,issue 8,August 2014 pp 1-12.

[13] Sundas Shahzadi, Muhammad Akram,Coloring of Bifuzzy Graph, Italian Journal of Pure and Applied Mathematics -N 36-2016(429-444). 


\section{Available online at www.ijrat.org}

[14] Yahya Mohamad S, Suganthi S.,Matching in Fuzzy Labeling Graph, International Journal of Fuzzy mathematical Archieve, vol14,No1,2017,155-161.

[15] Yahya Mohamad S, Suganthi S.Properties of Fuzzy Matching in Set Theory,Journal of Emerging Technologies and Innovative Research, Vol 5,issue2,july 2018.

[16] Yahya Mohamad S. Suganthi S.Operations in Fuzzy Labeling Graph through Matching and Complete Matching, International Journal of Applied Engineering Research vol 13 no 12(2018).PP10389-10393.

[17] Yahya Mohamad S. Suganthi S. Energy of Complete Fuzzy Labeling Graph through Fuzzy Complete Matching, International Journal of Mathematics Trends and Technology, Vol58,issue3-June 2018.

[18] Yahya Mohamad S, Suganthi S.Matching and Complete Matching Domination in Fuzzy Labeling Graph ,Journal of Applied Science and Computations, Vol5, Issue10, Oct 2018.

[19]Zadeh, L. A. Fuzzy sets, Information and Control 8 (1965), 338-353.

[20]Zepeng Li,Zehui Shao, Enqiang Zhu,Jin Xu,A Note On Local Coloring of Graphs, Information Processing Letter 115 (2015). 